\title{
ON THE ESTIMATION OF STRUCTURAL HEDONIC PRICE MODELS
}

\author{
By JAMES N. Brown ANd HARvey S. Rosen' ${ }^{1}$
}

\section{INTRODUCTION}

MANY COMmodrties can be viewed as bundles of individual attributes for which no explicit markets exist. It is often of interest to estimate structural demand and supply functions for these attributes, but the absence of directly observable attribute prices poses a problem for such estimation. In an influential paper published several years ago, Rosen [3] proposed an estimation procedure to surmount this problem. This procedure has since been used in a number of applications (see, for example, Harrison and Rubinfeld [2] or Witte, et al. [4]). The purpose of this note is to point out certain pitfalls in Rosen's procedure, which, if ignored, could lead to major identification problems.

In Section 2 we summarize briefly the key aspects of Rosen's method as it has been applied in the literature. Section 3 discusses the potential problems inherent in this procedure and provides an example. Section 4 concludes with a few suggestions for future research.

\section{ROSEN'S [3] PROCEDURE}

For convenience, we retain Rosen's notation. Suppose that some good is composed of $n$ attributes, $Z_{1}, \ldots, Z_{n}$. In general, the price of this good will depend on the quantities of the various attributes of which it is composed; hence one can express this price as some function $P(Z)$. Further, by differentiating $P(Z)$ with respect to its $i$ th argument, $Z_{i}$, one can derive the market equilibrium price function for $Z_{i}, P_{i}(Z)$, that is implicit in $P(Z)$.

Now, assume that consumers' marginal willingness to pay for $Z_{i}$ is some function $F_{i}(\cdot)$ of the quantities of all attributes as well as a vector of exogenous shift variables, $Y_{1}$. Similarly, assume the marginal supply price of $Z_{i}$ can be written as $G_{i}\left(Z, Y_{2}\right)$, where $Y_{2}$ is a vector of exogenous supply shift variables.

Interpreting $P_{l}(Z)$ as the (implicit) market price for attribute $Z_{l}$, the usual equilibrium assumptions lead to the following model to be estimated:

$$
\begin{aligned}
& P_{i}(Z)=F_{i}\left(Z_{1}, \ldots, Z_{n}, Y_{1}\right) \quad \text { (demand), } \\
& P_{i}(Z)=G_{i}\left(Z_{1}, \ldots, Z_{n}, Y_{2}\right) \quad \text { (supply), }
\end{aligned}
$$

for $i=1, \ldots n$. In this model, there are $2 n$ equations to determine the $2 n$ exogenous variables $P_{1}, \ldots, P_{n}, Z_{1}, \ldots, Z_{n}$.

Rosen suggests the following two-stage estimation procedure:

\begin{abstract}
"First, estimate $P(Z)$ by the usual hedonic method, without regard to $Y_{1}$ and $Y_{2}$. That is, regress observed differentiated products' prices, $P$, on all their characteristics. $Z$, using the best fitting functional form. This econometrically duplicates the information acquired by agents in the market, on the basis of which they make their decisions. Denote the resulting estimate of the function $P(Z)$ by $\hat{P}(Z)$. Next, compute a set of implicit marginal prices. $\partial P(Z) / \partial Z_{1}=\hat{P}_{i}(Z)$ for each buyer and seller evaluated at the amounts of characteristics
\end{abstract}

\footnotetext{
'Part of Rosen's work on this paper was supported by the National Science Foundation. We are grateful to John Ham. Richard Quandt, an editor and two referees for useful comments. An extended version of this paper is available as a memorandum with the same title issued by the Econometric Research Program. Princeton University.
} 
(numerical values of $Z$ ) actually bought or sold, as the case may be. Finally. use estimated marginal prices $\hat{P}_{i}(Z)$ as endogenous variables in the second-stage stimultaneous estimation of equations [1] and [2]. Estimation of marginal prices plays the same role here as do direct observations on prices in the standard theory and converts the second stage estimation into a garden variety identification problem." (Our emphasis.)

\section{SOME PITFALLS}

Contrary to Rosen's original statement, we claim that marginal attribute prices constructed as above will not necessarily play the same role in estimation that direct observations on prices would play if they were available. Because such constructed prices are created only from observed sample quantities, any new information that they may provide (i.e., any information beyond that already provided directly by observed sample quantities) can only come from a priori restrictions placed on the functional form of the price function $P(Z)$. In the absence of such additional restrictions, second-stage "structural" estimation of the sort suggested by Rosen may only reproduce the information already provided by the first-stage estimation of the $P(Z)$ function.

To see this point most simply, consider the following example. Suppose that the price function $P(Z)$ is second order in the $Z$ 's:

$$
P(Z)=\delta_{0}+\sum_{i=1}^{n} \delta_{i} Z_{i}+\sum_{i=1}^{n} \sum_{j=i}^{n} \delta_{i j} Z_{i} Z_{j}+u,
$$

where the $\delta$ 's are parameters and $u$ is an error term (observation subscripts have been suppressed for the sake of clarity). In this case, the marginal implicit price for $Z_{i}$ will be estimated by

$$
\hat{P}_{i}=\frac{\partial \hat{P}}{\partial Z_{i}}=\hat{\delta}_{i}+\sum_{j \neq i} \hat{\delta}_{i j} Z_{j}+2 \hat{\delta}_{i i} Z_{i}
$$

where "s denote estimated values.

Suppose further that the (inverse) demand and supply functions are linear in the $Z$ 's. Then, following Rosen's procedure, the equations to be estimated will be

$$
\begin{aligned}
& \hat{P}_{k}=\gamma_{k 0}+\sum_{i=1}^{n} \gamma_{k i} Z_{i}+\gamma_{k} Y_{1}+\omega_{k} \quad \text { (demand), } \\
& \hat{P}_{k}=\phi_{k 0}+\sum_{i=1}^{n} \phi_{k i} Z_{i}+\phi_{k} Y_{2}+\epsilon_{k} \quad \text { (supply), }
\end{aligned}
$$

for $k=1, \ldots n$, where $\omega_{k}$ and $\epsilon_{k}$ are random errors ${ }^{2}$ and the $\gamma$ 's and $\phi$ 's are structural parameters to be estimated.

In this particular example, Rosen's procedure will fail to identify the structural demand and supply functions that are of key interest. Indeed, the $\hat{\gamma}$ 's and $\hat{\phi}$ 's that emerge will be simple functions of the first stage $\hat{\delta}$ 's, and can be derived exactly without doing any second stage estimation at all. This fact is easily seen by looking at the ordinary least squares estimator for $\gamma_{k i}$ in (5).

$$
\hat{\gamma}_{k i}(\mathrm{OLS}) \equiv \frac{S_{\hat{P}_{h} \cdot Z_{i}}}{S_{Z_{i} \cdot Z_{i}}}
$$

\footnotetext{
${ }^{2}$ In this paper, we do not investigate the implications of alternative error structures in (5) and (6). For such a discussion, see Epple [1]
} 
where $S_{\hat{P}_{k} \cdot Z_{i}}$ denotes the sample partial covariance of $\hat{P}_{k}$ and $Z_{i}$ (i.e., the sample covariance of those elements of $\hat{P}_{k}$ and $Z_{i}$ that are uncorrelated with all other $Z$ 's and $Y_{1}$ ) and where $S_{Z_{i} \cdot Z_{i}}$ denotes analogously the sample partial variance of $Z_{i}$. Noting from (3) that $S_{\hat{P}_{k} \cdot Z_{i}}=\hat{\delta}_{k i} S_{Z_{i} \cdot Z_{i}}$, it can be seen immediately that applying ordinary least squares to equation (5) will not provide estimates of the $\gamma_{k i}$, but rather will simply yield various functions of the previously estimated parameters of equation (1). Specifically, the coefficient on $Z_{i}$ will be $2 \hat{\delta}_{i i}$, and that on $Z_{j}$ will be $\hat{\delta}_{i j}$.

This argument applies identically to the estimation of equation (6). Given that $\hat{P}_{k}$ is an exact function of the $Z$ 's, the presence of $Y_{2}$ rather than $Y_{1}$ in equation (6) will have no effect on the ratio in (7), and thus OLS estimation of equation (6) will lead to the same estimated coefficients for the Z's as produced by OLS estimation of equation (5), none of which reflects the structural parameters $\gamma_{k i}$ and $\phi_{k i}$. These results remain unchanged when (5) and (6) are estimated by two-stage least squares or, more generally, any instrumental variables method. ${ }^{3}$

The fundamental point illustrated by this example is that marginal "prices" constructed only from quantities do not in themselves add any information to that already provided by observations on quantities. The source of any additional information provided by such prices must lie in the a priori restrictions placed on the manner in which observed quantities are translated into marginal prices. Thus, even abstracting from standard structural identification issues, implementation of Rosen's procedure requires prior restrictions on functional form.

In general, problems of the sort that arise in the preceding example can be avoided only if the marginal price function for $Z_{i}$ cannot be expressed as some exact combination of the arguments of the structural demand or supply functions. If, for example, the market locus were assumed to be $m$ th order in the $Z$ 's, then the marginal price function for $Z_{i}$ would be of order $m-1$ in the $Z$ 's, and so long as the demand and supply functions for $Z_{i}$ were assumed to be of order $m-2$ or less in the $Z$ 's, second-stage estimation of (5) and (6) would not merely duplicate information from the market locus. ${ }^{4}$ The structural interpretation of the resulting estimates, of course, would depend upon the validity of the underlying restrictions.

Alternatively, if marginal prices were first estimated from equations fit separately for spatially distinct markets and then used to estimate a common underlying structure for all markets, simple reproduction of first-stage information again could be avoided. To elaborate, if observations were drawn from $r$ separate markets, then $r$ versions of the market locus (3) could be estimated, and for each observation, $P_{i}$ would depend not only upon the $Z$ vector associated with the observation, but also upon other market-specific

${ }^{3}$ PRoof: In this case. the estimator for $\gamma_{k}$ is

$$
\hat{\gamma}_{h i}(2 S L S)=\frac{S_{\hat{P}_{h} \cdot \dot{z}_{1}}}{S_{\dot{z}_{1} \cdot \dot{z}_{1}}}
$$

where $\hat{Z}_{1}$ denotes the first stage constructed instrument for $Z_{i}$, and where the variance and covariance notation remains as before. In this case, noting from (3) that $S_{\vec{P}_{k} \cdot \dot{Z}} \equiv \delta_{k i} S_{Z_{i} \cdot \dot{Z}}$, and recalling that, by construction, $S_{Z_{1} \cdot \dot{Z}_{1}}=S_{\dot{Z}_{1} \cdot \dot{Z}_{i}}$. it is once again seen that because of the particular identity relation linking $\hat{P}_{k}$ and the $Z$ 's, estimation of (5) and (6) using the constructed $\hat{P}_{k}$ will only reproduce the previously estimated parameters of (3). Finally, because the ratios in expressions (7) and (8) would be unchanged if the observed sample values of $\hat{P}_{k}$ and $\hat{Z}_{i}$ were first transformed by the same weighting scheme, using three-stage least squares rather than two-stage least squares or, more generally, using any generalized least squares procedure to estimate equations (5) and (6) would lead to the same results.

${ }^{4}$ Because any nonlinear function can be represented arbitrarily closely by a polynomial of some order, we consider only a polynomial market locus. 
factors reflected in the various coefficient estimates. If one were then willing to impose the condition that the structural demand and supply parameters be identical across markets, even though the hedonic price loci are not, then identification could be achieved. Of course, if there were not sufficient variation in the hedonic locus parameters across markets, nonsense estimates of (5) and (6) would nevertheless be obtained. In any case, unlike the garden variety simultaneous estimation problem, selection of appropriate shift variables is not the only key to identification of the structural parameters."

A number of these issues are exemplified in the work of Witte. Sumka, and Erekson [4]. who have applied Rosen's [3] technique to the study of housing markets. They specify a second order hedonic price equation like (3), and linear demand and supply equations like (5) and (6). Their hedonic price equation is estimated separately for four different cities. but the parameters of (5) and (6) are constrained to be identical across the cities. Although they do not note it, the fact that they use different cities is the only thing that
saves Witte, Sumka, and Erekson from estimating a series of identities in the second stage. ${ }^{6}$

\section{CONCLUSIONS}

We have shown the inappropriateness of assuming that equations like (5) and $(6)$ form a conventional simultaneous equation model withoul consideration of how the marginal attribute prices were generated from the sample data. When it is assumed that the prices are generated by a single hedonic price equation, the only way to identify the structural coefficients is by (possibly arbitrary) restrictions on functional form. When it is assumed that the prices are generated by several different equations, there may be sufficient "in-between" variation to generate meaningful structural parameters even without such constraints on functional forms. However, one must then impose the restriction that the structural parameters are identical across markets even though the hedonic locus parameters are not.

Although we have pointed out some pitfalls in the usual methods for estimating structural hedonic price models, we have not stated a general set of conditions under which identification is possible. Given the importance of hedonic models in applied research, the search for such conditions deserves serious attention.

\section{Princeton University}

Manuscript received August. 1980; revision received June, 1981.

5O course, the rank as well as order restrictions must be achieved.

"On the other hand. Harrison and Rubinfeld [2] avoid estimating an identity by assumptions on functional form.

\section{REFERENCES}

[1] Epptr. Drnnis: "Hedonic Prices and Implicit Markets: Estimating Demand and Supply Functions for Differentiated Products." mimeo, Carnegie-Mellón University. August. 1980.

[2] Harrison. David, and Danili. L. Rubinfrid: "Hedonic Housing Prices and the Demand for Clean Air." Journal of Environmental Economics and Management. 5(1978), 81-102.

[3] Rosfin. SHeRwin: "Hedonic Prices and Implicit Markets: Product Differentiation in Pure Competition." Journal of Political Economy. 82(1974). 34-55.

[4] Wittr. ann D. Howard J. Sumka. and Homer Erekson: "An Estimate of a Structural Hedonic Price Model of the Housing Market: An Application of Rosen's Theory of Implicit Markets." Econometrica, 47(1979). 1151-1173. 